\title{
EDITORIAL
}

\section{Should clinicians worry about vancomycin-resistant Enterococcus bloodstream infections?}

\author{
Bone Marrow Transplantation (2006) 38, 771-774. \\ doi:10.1038/sj.bmt.1705532
}

The clinical significance of vancomycin-resistant Enterococcus (VRE) infections has been debated for many years. An article in this issue of the journal by Dubberke and coworkers (Vancomycin-resistant enterococcal bloodstream infections on a hematopoietic stem cell transplant unit: are the sick getting sicker?) addresses this question. This is a retrospective cohort study of patients with hematologic malignancies and recipients of hematopoietic stem cell transplant (HSCT) who developed nosocomial VRE bloodstream infections (BSIs) over the course of almost 7 years at Barnes-Jewish Hospital. Data regarding VRE infections in this highly immunosuppressed population has been sparse, ${ }^{1,2}$ and this paper, reporting the characteristics and outcomes of 60 patients who suffered 68 VRE BSIs, represents the largest published series of VRE BSIs in this population to date. ${ }^{3}$

Infections with gram-positive bacteria are a major cause of morbidity in stem cell transplant recipients. Chemotherapy-induced neutropenia and mucositis, heavy exposure to prophylactic antimicrobials with limited gram-positive bacterial coverage and near universal use of central venous catheters predispose HSCT recipients to gram-positive bacteremia. Additionally, these patients frequently have had numerous hospitalizations with many courses of antibiotics that can promote the emergence and overgrowth of resistant colonizing flora. Lastly, because these patients are often seriously ill, they require extensive contact with health-care providers who through contamination of their hands, clothing and equipment represent the vector of transmission for resistant gram-positive organisms in health-care facilities. Taken together, these features place HSCT recipients at high risk for colonization and subsequent infection with resistant pathogens such as methicillin-resistant Staphylococcus aureus (MRSA) and VRE. $^{4}$

VRE was first described in the HSCT patient population in 1991..$^{5}$ Since then, there have been numerous reports documenting the increased rate of colonization of HSCT with VRE $(4.9-50 \%)^{6,7}$ and a subsequent rise in the frequency of VRE infections. ${ }^{8,9}$ These infections tend to occur early in the post-transplant period, typically before engraftment. ${ }^{2}$ Data on the clinical course of these infections has been conflicting with mortality rates attributed to VRE ranging from $\sim 10^{10}$ to $100 \%$. $^{2}$ A recent meta-analysis clearly documented that VRE, particularly in immunosuppressed patients, was independently associated with increased mortality among patients with enterococcal bloodstream infections. ${ }^{11,12}$

Also problematic is the lack of proven, safe and effective regimens in this population to treat VRE infections, which may contribute to a delay in the initiation of appropriate therapy. Additionally, many patients are treated with bacteriostatic regimens instead of bacteriocidal combinations because of concern for toxicity, which may also contribute towards the morbidity and mortality associated with VRE infections. A recently published study comparing the efficacy and safety of linezolid to that of vancomycin for use among febrile neutropenic patients with cancer revealed a delayed recovery of absolute neutrophil counts among those who had received linezolid; however, this phenomenon did not seem to interfere with recovery from bacterial infection. ${ }^{13}$ Furthermore, alternative agents such as daptomycin and quinupristin/dalfopristin have not been assessed for efficacy or safety in the immunosuppressed population. Studies are needed to better define the optimal management strategy of VRE infections in this unique population.

The study by Dubberke et al. ${ }^{3}$ provides some valuable epidemiologic information regarding the characteristics and outcomes of the patients who developed VRE BSI. Thirteen percent of patients known to be enterically colonized with VRE went on to develop a subsequent VRE BSI and remained bacteremic from 1 to 9 days. The majority of patients who suffered VRE BSI were acute leukemics, particularly those with relapsed or refractory disease. Additionally, among allograft recipients who developed infection, $42 \%$ had acute graft-versus-host disease (GVHD) at one or more sites and $32 \%$ had chronic GVHD. Other infections and antibiotic exposure were common preceding the VRE BSI and mucocutaneous barrier disruption was almost always present. These findings build on previous studies and refine our understanding of risk factors for VRE infection among the immunosuppressed.

The authors concluded that among patients with hematologic malignancies or HSCT, VRE may not have the behavior of a virulent pathogen and VRE BSI may simply be a marker of these patients' already existing critical medical condition. ${ }^{3}$ The evidence presented to support this conclusion was (1) the assessment that only four deaths among bacteremic patients were felt to be directly attributable to the VRE BSI, even though 32 patients with infection died, (2) there was no significant change in the APACHE II score before VRE BSI and within $48 \mathrm{~h}$ of VRE BSI to signify an acute clinical deterioration and (3) the fact that seven of 10 patients 
diagnosed with VRE BSI, who did not receive specific therapy directed towards their infection, did not develop recurrent VRE BSI (although three died within $48 \mathrm{~h}$ of acquiring their BSI).

The mortality rate attributable to VRE BSI in this study was $6.7 \%(4 / 60)$. Although lower than most studies assessing this outcome, some clinicians would consider this to be a worrisome finding that is clinically significant. Additionally, there was no discussion of the added morbidity and/or hospital cost associated with the VRE infections, nor was there a discussion regarding what the cause of death was attributable to among the other 28 patients who died. The study gives significant weight to the change in the APACHE II score, but does not take into account the fact that only $50 \%$ of patients were on appropriate antibiotics during the first $48 \mathrm{~h}$. If the rate of change of the APACHE II score was equal but in the opposite direction in these two groups, no difference would be seen if only the entire group was studied.

Factors found through multivariable analysis to be significantly associated with death among patients with VRE BSI in this study were age, hospitalization within the previous 30 days, Eastern Cooperative Oncology Group (ECOG) score $\geqslant 2$ on admission, transplant status, admission for GVHD, pneumonia, receipt of non-prophylactic anti-fungals or anti-anaerobic antibiotics, mechanical ventilation, acute neurologic dysfunction, total parenteral nutrition (TPN), APACHE II score, treatment of the VRE BSI with chloramphenicol and non-removal of the central venous catheter; however, if only 32 deaths occurred (the outcome of interest), some would argue that the multivariable analysis only had the power to assess three (or four at the most) variables in a reliable manner and some of these associations may have been seen by chance alone. ${ }^{14}$

The authors correctly state that the study had several limitations such as limited objective methods to control for complexity and severity of underlying illness, and the impact of effective therapy over time; however, the most disturbing limitation of the study is the lack of a control group, which makes any comparative statement regarding whether VRE BSI were more deadly among this patient population impossible. Without making comparisons to controls, one should not conclude, as the authors have, that 'VRE does not have the behavior of a virulent pathogen and that VRE BSI may simply be a marker of disease severity in patients at high-risk for mortality'. ${ }^{3}$

Lastly, an important finding of this study was the change in the rate of VRE BSI relative to the use of contact precautions (gowns and gloves) to care for patients colonized or infected with the organism. A program of surveillance, which included assessing for VRE colonization when stool was sent for Clostridium difficile testing, was also carried out. This surveillance and use of contact precautions began before the study period and the VRE BSI rate was maintained at 0.6-0.9 VRE BSI per 1000 patient days until 1998 when, because of cost concerns, this practice was abandoned and only gloves were required to care for VRE colonized or infected patients. This was followed by a significantly increased rate of VRE BSI to 2.1 per 1000 patient days. This increase prompted the reinstitution of the control program and the result was another significant decrease in the VRE BSI rate to 1.3 per 1000 patient days. This is perhaps one of the most important findings of this study that deserves further discussion.

The measures used to control resistant gram-positive organisms in hospitals have been debated for many years. Some would argue that conducting surveillance in order to identify patients colonized with resistant gram-positive pathogens and then caring for those patients with contact precautions is either too costly or simply does not work. Others would argue just the opposite: that a program of surveillance and the use of contact precautions can significantly decrease the rate of resistant gram-positive organisms (and thus infections) in a health-care facility in a cost-effective manner.

The most important reason why resistant gram-positive organisms have increased in many health-care facilities is that these organisms are spread from patient to patient via the transient contamination of health-care workers hands, ${ }^{15-17}$ apparel, ${ }^{16}$ personal equipment ${ }^{18}$ or medical equipment. ${ }^{16,19}$ Clinicians frequently do not wash their hands ${ }^{20}$ and almost never disinfect their equipment between patient contacts. Patients, with severe illness, who require more antibiotics and more intense nursing or physician contact, are particularly at risk for acquiring these pathogens. Even though prudent antibiotic use and increased hand hygiene rates likely will help, reducing patient to patient spread through the use of surveillance culturing to identify the colonized reservoir and contact precautions to care for those colonized has been repeatedly associated with control of resistant gram-positive organisms. $^{21}$ In a study which controlled for antimicrobial therapy, colonization pressure and spread from other colonized patients was the single most important predictor of a patient becoming colonized by $\mathrm{VRE}^{22}$ but, in another study, during a clonal VRE outbreak, being in close proximity to a VRE-colonized patient who was being cared for in contact isolation was not a risk factor for VRE acquisition. ${ }^{23}$ At least 12 published studies have described control of spread of endemic VRE, ${ }^{24-35}$ most without the use of antibiotic control as an adjunctive measure.

Multiple studies have documented higher morbidity, mortality and financial costs associated with nosocomial infections due to VRE than with infections due to susceptible strains of the same species, ${ }^{12,36}$ but some have questioned the cost-effectiveness of using surveillance cultures to identify and isolate all colonized patients. Studies assessing this for control of VRE have been conducted, each concluding that investing in a program to control spread by use of surveillance cultures and contact precautions was less expensive than taking no effective measures and allowing rates of VRE to continue to increase, ${ }^{25,35,37}$ usually because lower rates of infections were the ultimate outcome.

In conclusion, we are concerned that some who read this paper may conclude that VRE is not a significant pathogen and therefore does not warrant aggressive measures to identify and treat those who have it. The data provided only allows the conclusion that VRE may be a marker of severe illness but not that VRE is not associated with severe outcomes. Future studies with appropriate control groups 
would be necessary to address this issue. In the mean time, the majority of studies carried out to assess outcomes among patients with VRE infections would suggest that prolonged bacteremia, prolonged hospital stay and increased risk of death are common among those with VRE infections and clinicians should worry about VRE infections and strive to help control the spread of the organism.

CD Salgado ${ }^{1}$ and MG Ison ${ }^{2}$

${ }^{1}$ Department of Medicine, Division of Infectious Diseases, Hospital Epidemiologist, Medical University of South Carolina, Charleston, SC, USA and

${ }^{2}$ Division of Infectious Diseases, Transplant and Immunocompromised Host Infectious Diseases Service, Northwestern University Feinberg School of Medicine, Chicago, IL, USA

E-mail:salgado@musc.edu

\section{References}

1 Small $T$. Incidence and outcome of vancomycin-resistant enterococcal bacteremia following allogeneic hematopoietic stem cell transplant (HSCT). Abstracts of the 42nd Interscience Conference on Antimicrobial Agents and Chemotherapy (San Diego). American Society for Microbiology: Washington, DC, 2002, 325pp.

2 Avery R, Kalaycio M, Pohlman B, Sobecks R, Kuczkowski E, Andresen $\mathrm{S}$ et al. Early vancomycin-resistant Enterococcus (VRE) bacteremia after allogeneic bone marrow transplantation is associated with a rapidly deteriorating clinical course. Bone Marrow Transplant 2005; 35: 497-499.

3 Dubberke E, Hollands J, Georgantopoulos P, Augustin K, DiPersio J, Mundy L et al. Vancomycin-resistant enterococcal bloodstream infections on a hematopoietic stem cell transplant unit: are the sick getting sicker? Bone Marrow Transplant 2006; 38: 813-819.

4 Copelan E. Hematopietic stem-cell transplantation. $N$ Engl J Med 2006; 354: 1813-1826.

5 Peetermans W, Sebens F, Guiot H. Vancomycin-resistant Enterococcus faecalis in a bone-marrow transplant recipient. Scand J Infect Dis 1991; 23: 105-109.

6 Matar MJ, Safdar A, Rolston KV. Relationship of colonization with vancomycin-resistant enterococci and risk of systemic infection in patients with cancer. Clin Infect Dis 2006; 42: 1506-1507.

7 Tsiatis A, Manes B, Calder C, Billheimer D, Wilkerson K, Frangoul $\mathrm{H}$. Incidence and clinical complications of vancomycin-resistant enterococcus in pediatric stem cell transplant patients. Bone Marrow Transplant 2004; 33: 937-941.

8 Zinner S. Changing epidemiology of infections in patients with neutropenia and cancer on Gram-positive and resistant bacteria. Clin Infect Dis 1999; 29: 490-494.

9 Frere P, Hermanne J, Debouge M, Fillet G, Beguin Y. Changing pattern of bacterial susceptibility to antibiotics in hematopoietic stem cell transplant recipients. Bone Marrow Transplant 2002; 29: 589-594.

10 Kapur D, Dorsky D, Feingold J, Bona RD, Edwards RL, Aslanzadeh $\mathbf{J}$ et al. Incidence and outcome of vancomycinresistant enterococcal bacteremia following autologous peripheral blood stem cell transplantation. Bone Marrow Transplant 2000; 25: 147-152.

11 DiazGranados C, Jernigan J. Impact of vancomycin resistance on mortality among patients with neutropenia and enterococcal bloodstream infection. $J$ Infect Dis 2005; 191: 588-595.
12 DiazGranados C, Zimmer S, Klein M, Jernigan J. Comparison of mortality associated with vancomycin-resistant and vancomycin-susceptible enterococcal bloodstream infections: a metaanalysis. Clin Infect Dis 2005; 41: 327-333.

13 Jaksic B, Martinelli G, Perez-Oteya J, Hartman C, Leonard L, Tack K. Efficacy and safety of linezolid compared with vancomycin in a randomized, double-blind study of febrile neutropenic patients with cancer. Clin Infect Dis 2006; 42: 597-607.

14 Harrell F, Lee K, Califf R, Pryor D, Rosati R. Regression modeling strategies for improved prognostic prediction. Statist Med 1984; 3: 143-152.

15 Bhalla A, Pultz NJ, Gries DM, Ray AJ, Eckstein EC, Aron $\mathrm{DC}$ et al. Acquisition of nosocomial pathogens on hands after contact with environmental surfaces near hospitalized patients. Infect Control Hosp Epidemiol 2004; 25: 164-167.

16 Zachary KC, Bayne PS, Morrison VJ, Ford DS, Silver LC, Hooper DC. Contamination of gowns, gloves, and stethoscopes with vancomycin-resistant enterococci. Infect Control Hosp Epidemiol 2001; 22: 560-564.

17 Duckro AN, Blom DW, Lyle EA, Weinstein RA, Hayden MK. Transfer of vancomycin-resistant enterococci via health care worker hands. Arch Intern Med 2005; 165: 302-307.

18 Singh D, Kaur H, Gardner WG, Treen LB. Bacterial contamination of hospital pagers. Infect Control Hosp Epidemiol 2002; 23: 274-276.

19 Bernard L, Kereveur A, Durand D, Gonot J, Goldstein F, Mainardi JL et al. Bacterial contamination of hospital physicians' stethoscopes. Infect Control Hosp Epidemiol 1999; 9: 626-628.

20 Pittet D, Mourouga P, Perneger TV. Compliance with handwashing in a teaching hospital. Infection Control Program. Ann Intern Med 1999; 130: 126-130.

21 Muto CA, Jernigan JA, Ostrowsky BE, Richet HM, Jarvis WR, Boyce JM et al. SHEA guideline for preventing nosocomial transmission of multidrug-resistant strains of Staphylococcus aureus and enterococcus. Infect Control Hosp Epidemiol 2003; 24: 362-386.

22 Bonten MJ, Slaughter S, Ambergen AW, Hayden MK, van Voorhis J, Nathan C et al. The role of 'colonization pressure' in the spread of vancomycin-resistant enterococci: an important infection control variable. Arch Intern Med 1998; 158: $1127-1132$.

23 Byers KE, Anglim AM, Anneski CJ, Germanson TP, Gold HS, Durbin LJ et al. A hospital epidemic of vancomycinresistant Enterococcus: risk factors and control. Infect Control Hosp Epidemiol 2001; 22: 140-147.

24 Calfee DP, Giannetta ET, Durbin LJ, Germanson TP, Farr BM. Control of endemic vancomycin-resistant Enterococcus among inpatients at a university hospital. Clin Infect Dis 2003; 37: $326-332$.

25 Calfee DP, Farr BM. Infection control and cost control in the era of managed care. Infect Control Hosp Epidemiol 2002; 23 : 407-410.

26 Vernon M, Haydem M, Trick W, Hayes R, Blom D, Weinstein $\mathrm{R}$ et al. Chlorhexadine gluconate to cleanse patients in a medical intensive care unit. Arch Intern Med 2006; 166: 306-312.

27 Wright MO, Hebden JN, Harris AD, Shanholtz CB, Standiford HC, Furuno JP et al. Aggressive control measures for resistant Acinetobacter baumannii and the impact on acquisition of methicillin-resistant Staphylococcus aureus and vancomycin-resistant Enterococcus in a medical intensive care unit. Infect Control Hosp Epidemiol 2004; 25: 167-168.

28 Rubin LG, Tucci V, Cercenado E, Eliopoulos G, Isenberg HD. Vancomycin-resistant Enterococcus faecium in hospitalized children. Infect Control Hosp Epidemiol 1992; 13: 700-705. 
29 Montecalvo MA, Jarvis WR, Uman J, Shay DK, Petrullo C, Rodney $\mathrm{K}$ et al. Infection-control measures reduce transmission of vancomycin-resistant enterococci in an endemic setting. Ann Intern Med 1999; 131: 269-272.

30 Silverblatt FJ, Tibert C, Mikolich D, Blazek-D'Arezzo J, Alves $\mathrm{J}$, Tack $\mathrm{M}$ et al. Preventing the spread of vancomycin-resistant enterococci in a long-term care facility. J Am Geriatr Soc 2000; 48: $1211-1215$.

31 Siddiqui AH, Harris AD, Hebden J, Wilson PD, Morris Jr JG, Roghmann MC. The effect of active surveillance for vancomycin-resistant enterococci in high-risk units on vancomycin-resistant enterococci incidence hospital-wide. Am J Infect Control 2002; 30: 40-43.

32 Price CS, Paule S, Noskin GA, Peterson LR. Active surveillance reduces the incidence of vancomycin-resistant enterococcal bacteremia. Clin Infect Dis 2003; 37: 921-928.

33 Ostrowsky BE, Trick WE, Sohn AH, Quirk SB, Holt S, Carson LA et al. Control of vancomycin-resistant entero- coccus in health care facilities in a region. $N$ Engl J Med 2001; 344: 1427-1433.

34 Jochimsen EM, Fish L, Manning K, Young S, Singer DA, Baker $\mathrm{R}$ et al. Control of vancomycin-resistant enterococci at a community hospital: efficacy of patient and staff cohorting. Infect Control Hosp Epidemiol 1999; 19: 106-109.

35 Muto CA, Giannetta ET, Durbin LJ, Simonton BM, Farr BM. Cost-effectiveness of perirectal surveillance cultures for controlling vancomycin-resistant Enterococcus. Infect Control Hosp Epidemiol 2002; 23: 429-435.

36 Salgado CD, Farr BM. Outcomes associated with vancomycinresistant enterococci: a meta-analysis. Infect Control Hosp Epidemiol 2003; 24: 690-698.

37 Montecalvo MA, Jarvis WR, Uman J, Shay DK, Petrullo C, Horowitz HW et al. Costs and savings associated with infection control measures that reduced transmission of vancomycin-resistant enterococci in an endemic setting. Infect Control Hosp Epidemiol 2001; 22: 437-442. 